\title{
Amino multi-walled carbon nanotubes further improve the thermal conductivity of boron nitride/liquid crystal epoxy resin composites
}

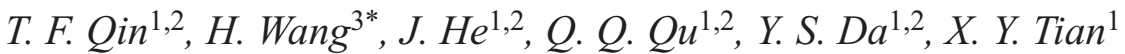 \\ ${ }^{1}$ Institute of Applied Technology, Hefei Institutes of Physical Science, Chinese Academy of Sciences, 230088 Hefei, \\ P. R. China \\ ${ }^{2}$ University of Science and Technology of China, 230026 Hefei, P. R. China \\ ${ }^{3}$ Hefei Institute of Technology Innovation, Chinese Academy of Sciences, 230031 Hefei, P. R. China
}

Received 2 April 2020; accepted in revised form 29 May 2020

\begin{abstract}
In this work, we introduced highly thermally conductive and fibrous amino multi-walled carbon nanotubes $\left(\mathrm{MCNT}-\mathrm{NH}_{2}\right)$ into hexagonal boron nitride/liquid crystal epoxy resin (h-BN/LCER) composites to improve the thermal conductivity of the composites. First, we prepared hexagonal boron nitride@amino multi-walled carbon nanotubes (h-BN@MCNT-NH $\mathrm{N}_{2}$ ) hybrid fillers. Then, the amino group in the hybrid filler participated in the curing process of the epoxy resin to prepare hexagonal boron nitride@amino multi-walled carbon nanotubes/liquid crystal epoxy resin (h-BN@MCNT$\mathrm{NH}_{2}$ /LCER) composites. Subsequently, its thermal conductivity was tested and analyzed using the Agari's model and microstructure of the composites, and we can come to conclude that the thermal conductivity of h-BN@MCNT-NH $/ \mathrm{LCER}$ composites is higher than that of $\mathrm{h}-\mathrm{BN} / \mathrm{LCER}$ at the same filler content. The main reason is that the addition of $\mathrm{MCNT}^{-\mathrm{NH}_{2}}$ plays a role in increasing the thermal conduction path of h-BN/LCER composites and decreasing the large interface thermal resistance of fillers and resin matrix. Finally, the usability and thermal conductivity of h-BN@MCNT-NH $/$ LECR composites were verified by light-emitting diode (LED) lamps. The temperature of LED lamp using 50\% h-BN@MCNT-NH $/$ LCER composites was eventually stabilized at $27.7^{\circ} \mathrm{C}$, it is expected that $50 \%$ h-BN@MCNT-NH $2 /$ LCER composites will be used in LED electronic products.
\end{abstract}

Keywords: polymer composites, thermal conductivity, amino multi-walled carbon nanotubes, hybrid filler, application in LED lamps

\section{Introduction}

In recent years, with the increasing miniaturization and multi-functionalization of electronic devices, the heat generated by integrated circuits during operation has also increased [1]. If the accumulated heat energy cannot be transferred in time, it will reduce the durability of the device $[2,3]$. The material with a good thermal conductivity can release easily the heat generated in the devices in order to maintain the temperature of the device at the desired level [4]. At present, many researchers are working to improve the thermal conductivity of polymer-based composites. Epoxy resin is often selected as a thermally conductive polymer matrix due to its good processing performance, high insulation performance, excellent mechanical properties, low water absorption, and outstanding adhesion force $[5,6]$. However, the thermal conductivity of epoxy resin is about $0.2 \mathrm{~W} /(\mathrm{m} \cdot \mathrm{K})$, which is far from the requirement of heat conduction in its electronic package [7, 8]. Generally, highly thermally conductive inorganic fillers such as hexagonal boron nitride (h-BN) [9], aluminum nitride(AlN) [10], 
silicon carbide ( $\mathrm{SiC})$ nanowires [11], graphene nanoplatelets (GNPs) [12] and carbon nanotubes (CNTs) [13] are added to the epoxy resin to increase the thermal conductivity. However, due to their high interface thermal resistance, the thermal conductivity of the final composite cannot be further improved [14]. Hexagonal boron nitride ( $\mathrm{h}-\mathrm{BN}$ ) is a two-dimensional layered material with excellent thermal conductivity [15]. Current theoretical researches show that its thermal conductivity can reach $600 \mathrm{~W} /(\mathrm{m} \cdot \mathrm{K})$ [16]. h-BN is a chemically inert material, which makes the interface bond with the polymer matrix weak, so its thermal conductivity cannot be well exhibited. Traditionally, h-BN is surface-modified by $\pi-\pi$ interaction and Lewis acid-base complexation between $\mathrm{h}-\mathrm{BN}$ and organic molecules modifier to reduce the interface thermal resistance between the filler and the polymer [18]. Wu and Kessler [14] non-covalently modified h-BN by the pi-pi interaction between $\mathrm{BN}$ and dopamine molecules and then added the modified h-BN into bisphenol E cyanate ester to prepared high-thermal conductivity h-BN/bisphenol E cyanate ester (h-BN/BECy) composites. Pan et al. [8] used KH550 modified h-BN to enhance the interfacial adhesion between h-BN and the matrix, and the thermal conductivity is improved compared with the unmodified h-BN composite. However, these modified methods cannot significantly improve the thermal conductivity of the composite $[17,19]$. At present, the thermal conductivity of polymer-based composites has not reached the ideal thermal conductivity, which is mainly due to the large interface thermal resistance of fillers and resin matrix [20].

Recently, carbon nanotubes (CNTs) garnered much attention due to intriguing electrical, thermal, and optical properties, as well as the large aspect ratio [21]. CNTs have a thermal conductivity of up to $3000 \mathrm{~W} /(\mathrm{m} \cdot \mathrm{K})$, which was several tens of times the thermal conductivity of conventional thermally conductive fillers [22]. Therefore, by placing it in a polymer, the thermal conductivity of the polymer-based composite can be significantly improved, which had been confirmed by other researchers. Shi and Zhu [23] fabricated silicone-modified acrylic resin-based (SiMA) nanocomposites. The surfaces of GNPs and CNTs were modified with $N, N^{\prime}$-(cyclohexa-2,5-diene1,4-diylidene)bis(2-hydroxy- $N$-(2-hydroxy-3-(4-(trihydroxysilyl)butoxy)propyl)-3-(4-(trihydroxysilyl) butoxy)propan-1-aminium) (TSiPD) as tGNPs and tCNTs. The tGNPs and tCNTs were added to Si-MA, becoming Si-MA nanocomposites. The thermal conductivity with $10 \mathrm{wt} \%$ tGNPs obtains $2.31 \mathrm{~W} /(\mathrm{m} \cdot \mathrm{K})$. It is worth noting that a high thermal conductivity was obtained $(2.97 \mathrm{~W} /(\mathrm{m} \cdot \mathrm{K}))$ with $9.9 \mathrm{wt} \% \mathrm{tGNPs}$ and $0.1 \mathrm{wt} \%$ tCNTs, which corresponds to a $28.5 \%$ increase compared to the value of $10 \mathrm{wt} \%$ tGNPs. Kim et al. [24] investigated the thermal conductivity of multi-walled carbon nanotube-embedded cyclic butylene terephthalate thermoplastic nanocomposites. Thermal conductivity test results show that the thermal conductivity of nanocomposites gradually increases with the increase of carbon nanotubes. Guo et al. [25] prepared triethoxyvinylsilane (YDH-151) functionalized multi-wall carbon nanotubes (s-MWCNTs) and blended into PVDF to achieve high thermal conducive s-MWCNTs/PVDF nanocomposites. Thermal conductivity of $1.552 \mathrm{~W} /(\mathrm{m} \cdot \mathrm{K})$ was achieved in s-MWCNTs/PVDF composite with $10 \mathrm{wt} \% \mathrm{~s}-\mathrm{MWCNTs}$ loading, about 9 times in comparison to that of pure PVDF matrix. In order to largely improve thermal conductivity, $\mathrm{CNT}-\mathrm{NH}_{2}$ were usually used. Yan et al. [26] research indicates that CNT- $\mathrm{NH}_{2}$ can participate in the curing of epoxy resin. Therefore, if the sheet-shaped h-BN and the fibrous $\mathrm{CNT}-\mathrm{NH}_{2}$ form a hybrid filler, it can improve the thermal conduction path and reduce the thermal resistance of the interface between the filler and the matrix, thereby improving the thermal conductivity of the composite material.

In this work, we introduced highly thermally conductive and fibrous multi-walled amino carbon nanotubes (MCNT-NH${ }_{2}$ ) into hexagonal boron nitride/ liquid crystal epoxy resin (h-BN/LCER) composites to improve the thermal conductivity of the composites. First, we prepared h-BN@MCNT-NH $\mathrm{N}_{2}$ hybrid fillers. Then, the amino group in the hybrid filler participated in the curing process of the epoxy resin to prepare h-BN@MCNT-NH $\mathrm{N}_{2} / \mathrm{LCER}$ composites [26]. $\mathrm{MCNT}-\mathrm{NH}_{2}$ plays a role in decreasing the large interface thermal resistance of fillers and resin matrix, thereby improving the thermal conductivity of h-BN/ LCER composites.

\section{Experiment}

\subsection{Materials}

A representative liquid crystal epoxy resins (LCERs) was selected, which is composed of epoxy monomer (3,3',5,5'-tetramethyl-4,4'-biphenyl diglycidyl ether (TMBPDGE)) and curing agent $\left(4,4^{\prime}\right.$-diaminodiphenylmethane (DDM)). 3,3',5,5'-tetramethyl-4,4'- 
biphenyl diglycidyl ether (TMBPDGE, YX-4000) were purchased from Shanghai Zhongsi Industrial Co., Ltd., China. The values of epoxy equivalent weight (EEW) of TMBPDGE were 185 g/eq. 4,4'diaminodiphenylmethane (DDM, purity $>97 \%$ ) were provided by Aladdin Industrial Co., Ltd., China. Hexagonal boron nitride platelets (h-BN, diameter $=$ $15 \mu \mathrm{m}$, purity $>99.5 \%$ ) were obtained from Dandong Rijin Science and Technology Co., Ltd., China. amino multi-walled carbon nanotube (MCNT-NH ${ }_{2}$, length $=50 \mu \mathrm{m}$, diameter $=8-15 \mathrm{~nm}$, purity $>95 \%$, $-\mathrm{NH}_{2}>0.45 \%$ ) were obtained from Aladdin Industrial Co., Ltd., China. Ethanol (humidity $<0.2 \%$, purity $>99.5 \%$ ) was purchased from Aladdin Industrial Co., China.

\subsection{Preparation of hybrid filler h-BN@MCNT-NH}

Figure 1a shows the process of surface treatment of h-BN. The h-BN $(5 \mathrm{~g})$ was dispersed in $50 \mathrm{ml}$ ethanol, and then $0.2 \mathrm{~g}$ of $\mathrm{MCNT}-\mathrm{NH}_{2}$ was added. The mixture was loaded inside a $500 \mathrm{ml}$ agate milling container containing $150 \mathrm{~g}$ of agate balls $(8 \mathrm{~mm}: 5 \mathrm{~mm}: 3 \mathrm{~mm}=3: 2: 1)$, The mixtures were milled at a rotation speed of $300 \mathrm{rpm}$ for $3 \mathrm{~h}$, and then the obtained product was dried in an oven at
$60{ }^{\circ} \mathrm{C}$ for $24 \mathrm{~h}$. The final material is named as h-BN@MCNT-NH $\mathrm{N}_{2}$, and the ratio of MCNT-NH $\mathrm{N}_{2}$ is 25 to 1 .

\subsection{Preparation of h-BN@MCNT-NH $/$ LCERs}

In this work, h-BN@MCNT-NH $2 /$ LCER composites were fabricated, as shown in Figure 1b. First, a certain amount of hybrid filler h-BN@ MCNT-NH $\mathrm{M}_{2}$ (h-BN: MCNT-NH ${ }_{2}=25: 1$ ) is ground together with DDM for 10 min to eliminate hydrogen bonds between DDM molecules. Second, TMBPDGE was placed in the mixture while grinding for $10 \mathrm{~min}$. The mixture of uncured TMBPDGE-DDM with the fillers was poured into the silicone oil paper, melt-blended, and precured at $160^{\circ} \mathrm{C}$ for $10 \mathrm{~min}$. The pre-cured sample was hot-pressed for $4 \mathrm{~min}$, then cured in an oven at $160^{\circ} \mathrm{C}$ for 7 h. Finally, h-BN@MCNT-NH $/ \mathrm{HCERs}_{2}$ was prepared. In the same way, h-BN/LCER composites with different filler contents were prepared, in which the mass fraction of boron nitride was 10, 20, 30, 40, and $50 \%$, and the corresponding volume fraction was $5.67,11.67,18.2,25.84$, and $35.32 \mathrm{vol} \%$. The composition of h-BN@MCNT-NH $2 /$ LCERs composites with different contents showed in Table 1. All prepared composites will be tested in the future.

Table 1. The composition of h-BN@MCNT-NH$/ 2$ LCERs composites with different contents.

\begin{tabular}{|c|c|c|c|c|}
\hline Sample code & $\begin{array}{c}\text { LCER } \\
{[\mathrm{g}]}\end{array}$ & $\begin{array}{c}\text { h-BN@MCNT-NH } \\
{[\mathrm{g}]}\end{array}$ & $\begin{array}{c}\text { h-BN@MCNT-NH } \\
{\left[w H_{2}\right.} \\
{[w]}\end{array}$ & $\begin{array}{c}\text { h-BN@MCNT-NH } \\
\text { [vol\%] }\end{array}$ \\
\hline LCER (TMBPDGE-DDM) & 6.492 & 0.000 & 0 & 0.00 \\
\hline 10\% h-BN@MCNT-NH $2 /$ LCERs & 6.492 & 0.721 & 10 & 5.61 \\
\hline 20\%íh-BN@MCNT-NH $2 /$ LCERs & 6.492 & 1.623 & 20 & 12.15 \\
\hline 30\% h-BN@MCNT-NH $2 /$ LCERs & 6.492 & 2.782 & 30 & 19.07 \\
\hline 40\% h-BN@MCNT-NH $2 /$ LCERs & 6.492 & 4.328 & 40 & 27.17 \\
\hline $50 \%$ h-BN@MCNT-NH $2 /$ LCERs & 6.492 & 6.492 & 50 & 36.57 \\
\hline
\end{tabular}

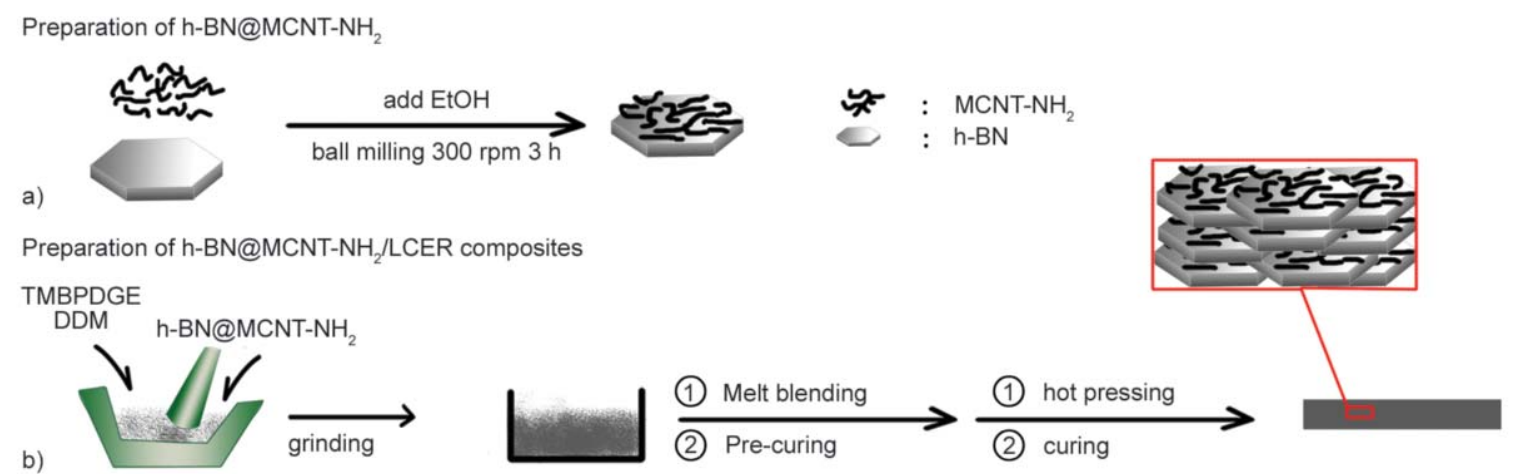

Figure 1. Schematic illustration of the procedure to prepare the composites. (a) Preparation of h-BN@MCNT-NH $\mathrm{N}_{2}$; (b) Preparation of h-BN@MCNT-NH $2 /$ LCER composites. 


\subsection{Characterization}

Raman spectra were measured by a Confocal Raman Microscopy (Renishaw in Via Reflex, Renishaw company, UK) with an excitation wavelength of $514.5 \mathrm{~nm}$. The X-ray film and powder diffractometer (XRD, Rigaku SmartLab SE, Rigaku Corporation, Japan) was used to record the orientation behavior of the composite with a high-speed detector D/teXUltra. The morphologies of the fillers and composites were characterized via scanning electron microscopy (SEM, Sirion-200, FEI, USA) in an accelerating voltage of $10 \mathrm{kV}$. The thermal conductivities of composites $(25 \mathrm{~mm} \times 25 \mathrm{~mm} \times 2 \mathrm{~mm})$ were tested on a Hot Disk instrument (TPS 2200, AB Corporation, Sweden). Thermal properties of composites were characterized using a TGA ( TG 209F3, NETZSCH, Germany) from 50 to $700^{\circ} \mathrm{C}$ at a heating rate of $10^{\circ} \mathrm{C} / \mathrm{min}$ under nitrogen condition. Self-installed components verify the possibility of composite materials applied in the field of electronic packaging. The devices are $3 \mathrm{~W}$ LED lamps; Infrared camera
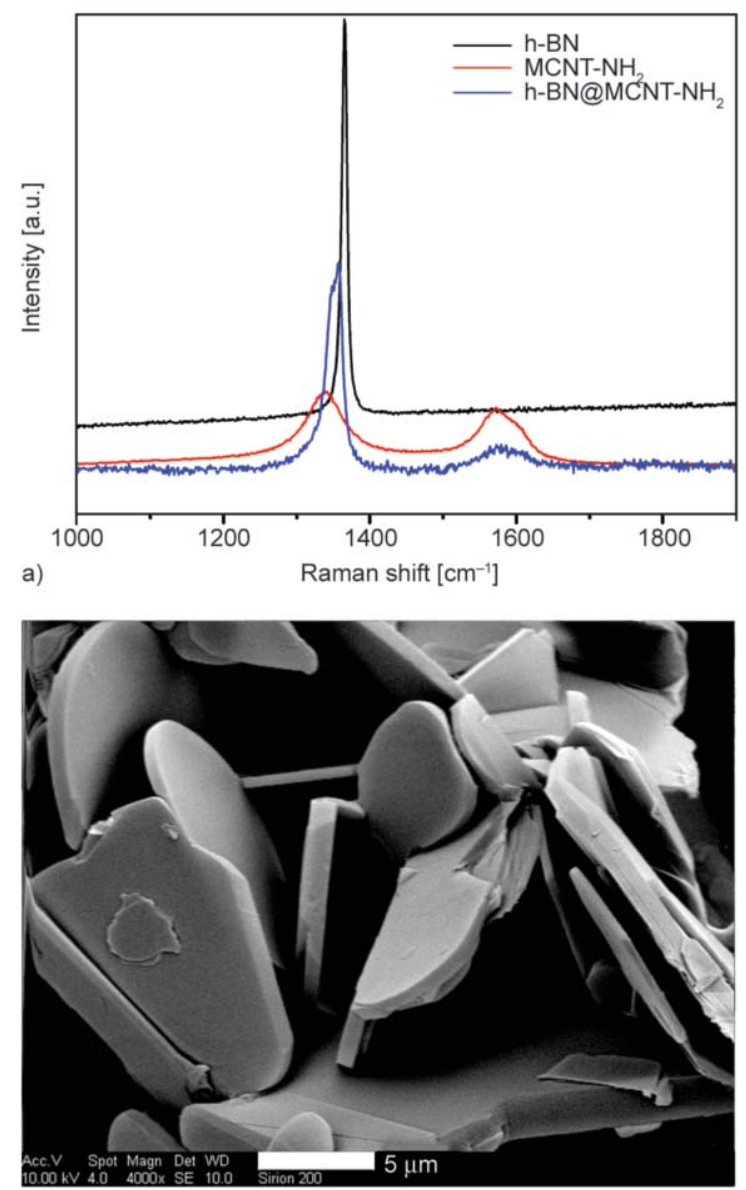

c)
(CEM DT-980, Shenzhen Huashengchang Technology Industrial Co., Ltd., China) was used to record the temperature change of the composite material.

\section{Results and discussion}

\subsection{Characterization of h-BN@MCNT-NH}

The preparation process of hybrid filler h-BN@MCNT-NH $\mathrm{N}_{2}$ was shown in Figure 1. Ethanol was used as solvent to improve the dispersibility of h-BN platelets and prevent aggregation of MCNT$\mathrm{NH}_{2}$ with each other. After h-BN and MCNT-NH were milled at a rotation speed of $300 \mathrm{rpm}$ for $3 \mathrm{~h}$, hybrid filler h-BN@MCNT-NH $\mathrm{N}_{2}$ was prepared. In Su et al. [27], the hybrid filler h-BN@CNT-NH $\mathrm{N}_{2}$ was prepared by ultrasonic treatment, using the $\pi-\pi$ interaction force between $\mathrm{h}-\mathrm{BN}$ and $\mathrm{CNT}-\mathrm{NH}_{2}$. It can be found from the morphology SEM images of h-BN@CNT-NH ${ }_{2}$ in that paper that CNT-NH ${ }_{2}$ on the surface of h-BN is easier to agglomerate. We used the method of ball milling to prepare h-BN@MCNT$\mathrm{NH}_{2}$, which can make the carbon tube adhere to the
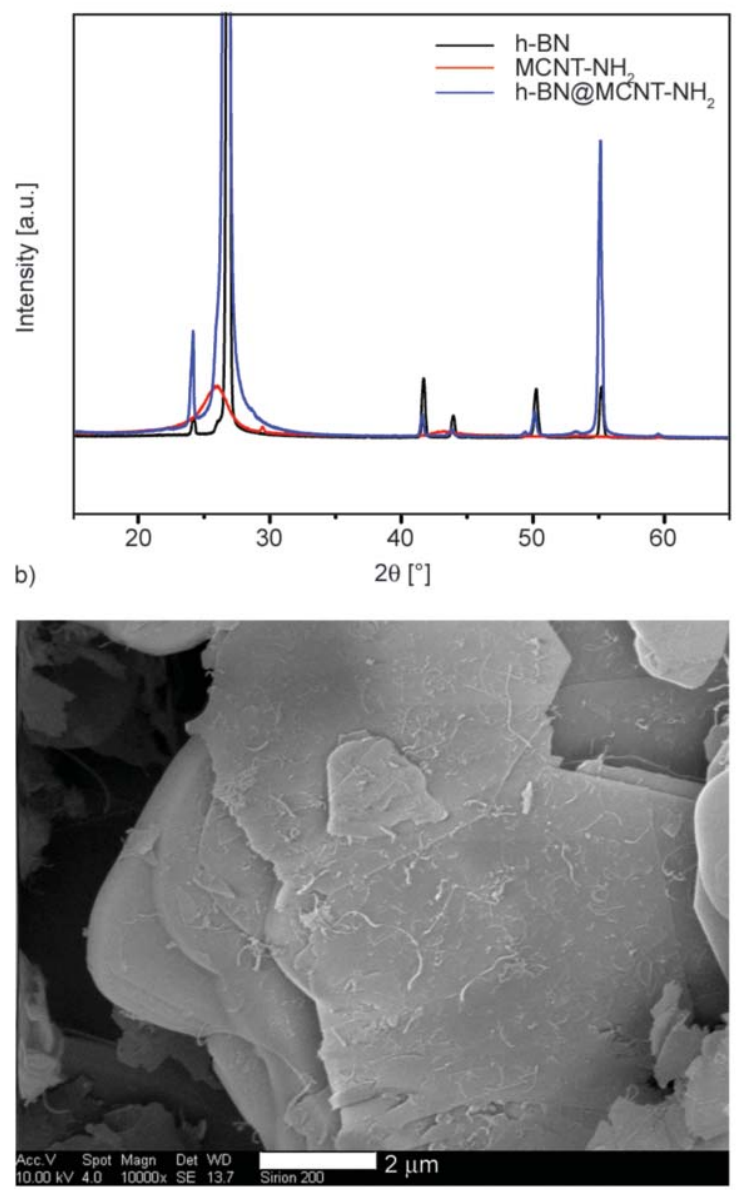

d)

Figure 2. (a) Raman spectra and (b) XRD patterns of pristine h-BN, $M C N T-\mathrm{NH}_{2}$, and h-BN@MCNT-NH $\mathrm{NH}_{2}$ ( (c) and (d) are the micromorphology of pure h-BN and hybrid filler h-BN@MCNT-NH $\mathrm{N}_{2}$ respectively. 
surface of boron nitride more easily and disperse more uniformly (Figure 2d). There are two possible reasons. On the one hand, the $\pi-\pi$ interaction between $\mathrm{BN}$ and MCNT-NH $\mathrm{N}_{2}$ is formed. On the other hand, due to the ball milling process, the surface of boron nitride has a certain roughness, which is easier to adhere to the carbon tube. Raman spectra of h-BN, MCNT-NH $\mathrm{N}_{2}$, and h-BN@MCNT-NH $\mathrm{N}_{2}$ were presented in Figure 2a. The characteristic peak of h-BN appeared at $1365 \mathrm{~cm}^{-1}$, which belonged to a Raman $G$ band due to the high frequency E2g vibration mode [28]. The $\mathrm{G}$ band was downshifted in the h-BN@MCNT-NH ${ }_{2}$ composite from 1365 to $1357 \mathrm{~cm}^{-1}$ compared to that of pristine h-BN, this effect was caused by the strong specific interactions between h-BN and MCNT-NH ${ }_{2}$ [29]. This interaction may be due to the $\pi-\pi$ effect between h-BN and MCNT-NH $\mathrm{N}_{2}$ and the rough surface the h-BN formed during ball milling, easy to combine $\mathrm{h}-\mathrm{BN}$ and MCNT-NH ${ }_{2}$.

To confirm adsorption of the MCNT-NH $\mathrm{N}_{2}$ on the surface of h-BN, X-ray diffraction (XRD) measurements were taken at $25^{\circ} \mathrm{C}$ to h-BN, MCNT-NH $\mathrm{N}_{2}$, and h-BN@MCNT-NH 2 . As showed in Figure 2b, a characteristic diffraction peak of h-BN was observed at $26.86^{\circ}$ corresponding to 100 crystal planes [30]. In addition, the (002) peak slightly shifted from $26.86^{\circ}$ (pristine h-BN) to $26.80^{\circ}$ after hybrid filler h-BN@MCNT-NH 2 was made. The possible reason for the peak downshift is that the ball milling mixing promotes the $\pi-\pi$ combination of $h-B N$ and MCNT-NH ${ }_{2}$. The microstructures of h-BN and h-BN@MCNT-NH $\mathrm{N}_{2}$ were observed through SEM (Figure 2c, 2d). By comparing pure h-BN with h-BN@MCNT-NH ${ }_{2}$, it can be seen that MCNT-NH are uniformly attached to the $\mathrm{BN}$ surface.

\subsection{Morphology of h-BN@MCNT-NH $/$ LECRs}

After pre-curing the composite, it was placed in a $3 \times 3 \times 0.2 \mathrm{~cm}$ mold, hot-pressed, and then cured in an oven to form h-BN@MCNT-NH $/$ LECR composites. Cross-sectional images of h-BN@MCNT- $\mathrm{NH}_{2} /$ LECR were characterized via SEM. As shown in Figure 3 , the dispersion and orientation of different content of fillers in the polymer can be visually observed. $\mathrm{h}-\mathrm{BN}$ is relatively uniformly dispersed in the composite. With increasing the loading of filler, the horizontal structure of the thermally conductive filler is regularly arranged. The reason for the increase of h-BN arrangement is that the pressure of the hot-pressed machine does not change when the content of the filler is high, the pressure is applied to make the h-BN plates squeeze each other laterally, thereby allowing the plates to be regularly aligned horizontally.

In order to further analyze the microstructure of 50 wt $\%$ h-BN@MCNT-NH $2 /$ LECRs, the distribution of filler the composite was observed at SEM (Figure $4 b$ ) using high magnification. MCNT- $\mathrm{NH}_{2}$ is slightly agglomerated because the carbon tube is introduced into the composite material through the hybrid filler. We chose 50\% h-BN/LCERs for comparison, as shown in Figure 4a, the h-BN filler is dispersed in h-BN/LCER horizontally, and the surface of epoxy matrix is smooth. The low thermal conductivity of $50 \mathrm{wt} \% \mathrm{~h}-\mathrm{BN} / \mathrm{LCERs}$ compared to 50 wt $\%$ h-BN@MCNT-NH $/$ LECRs is attributed to thermal energy to be consumed in the composite, which is harmful to conduct thermal energy (Figure 4c). It is noted that the epoxy resin matrix at our prepared h-BN@MCNT-NH $/$ LCERs contains carbon nanotubes in Figure $4 \mathrm{~b}$. The main reason might be that the MCNT- $\mathrm{NH}_{2}$ on the surface of h-BN

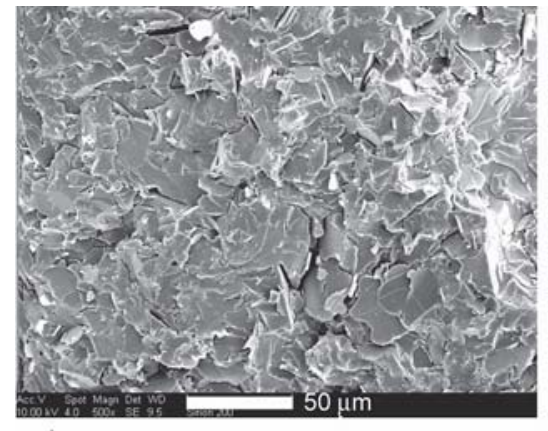

a)

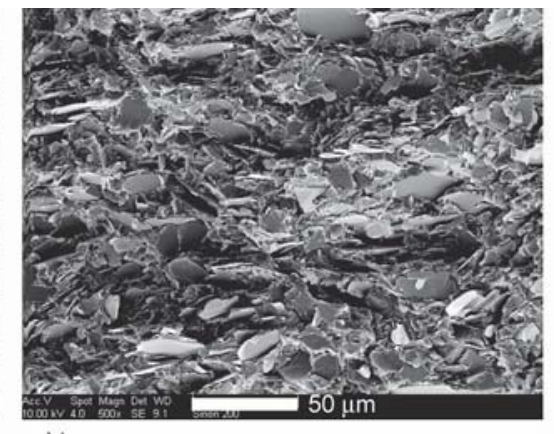

b)

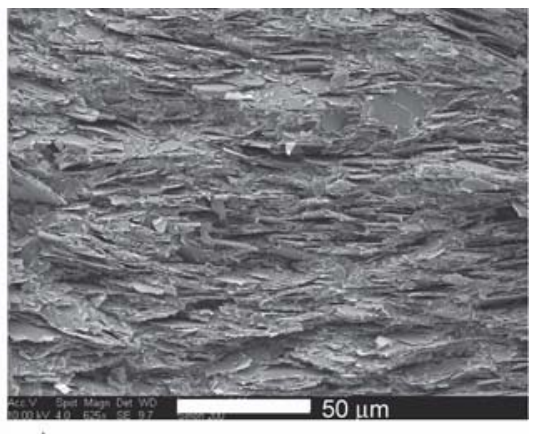

c)

Figure 3. Cross-sectional SEM images of h-BN@MCNT- $\mathrm{NH}_{2} / \mathrm{LECRs}$ with the different content of fillers (a) 10\% h-BN@MCNT-NH 2 LECRs; (b) 30\% h-BN@MCNT-NH $/$ LECRs; (c) 50\% h-BN@MCNT-NH $2 /$ LECRs. 


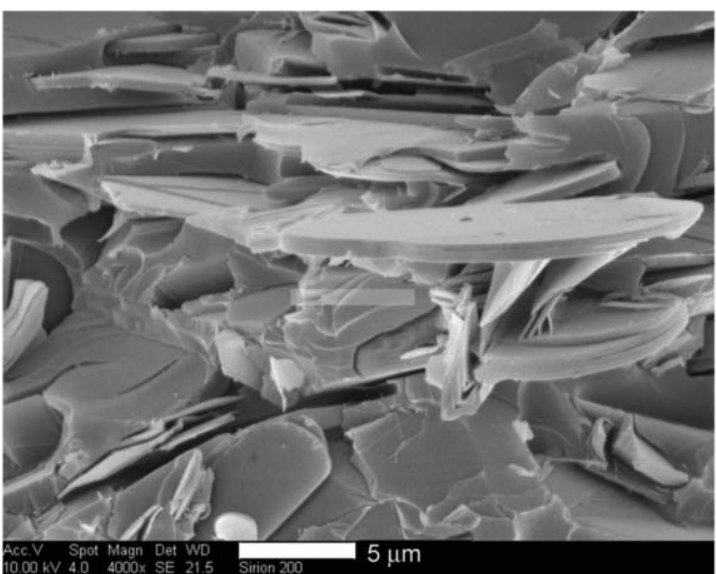

a)

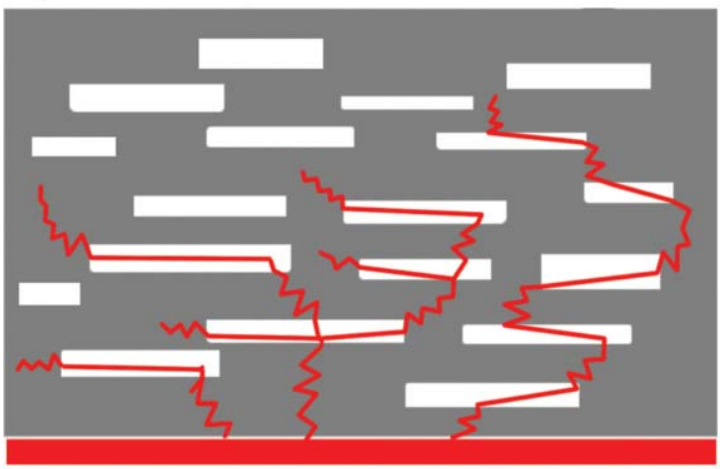

c)

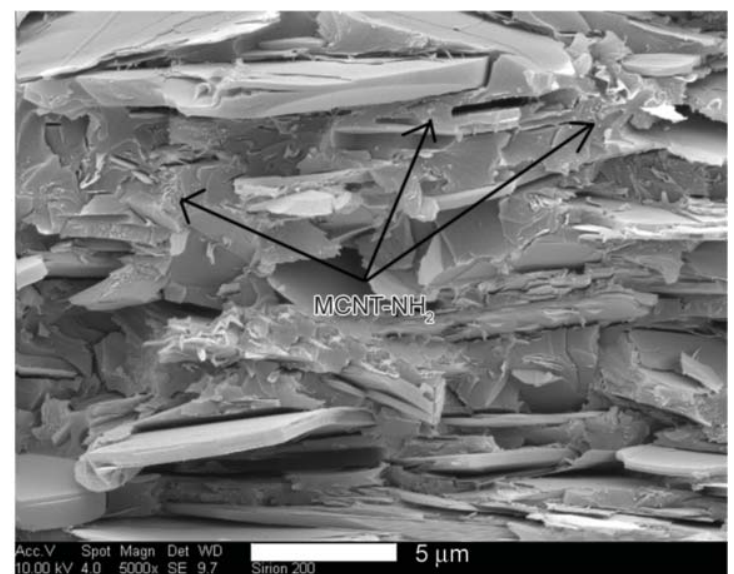

b)

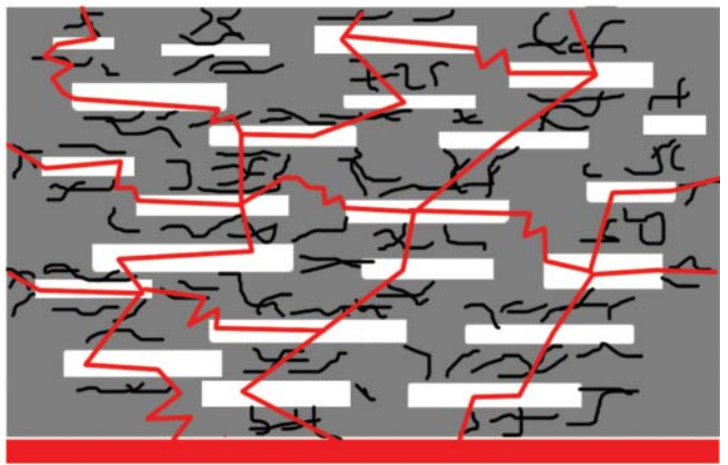

d)

Figure 4. (a) The microstructure of the 50\% h-BN/LECRs. (b) the microstructure of 50\% h-BN@MCNT-NH $/ 2$ LECRs. (c) and (d) are heat dissipation simulations of 50\% h-BN/LCERs and 50\% h-BN@MCNT-NH $2 /$ LCERs composites, respectively.

platelets participated in the epoxy resin curing process, which the amino group in MCNT- $\mathrm{NH}_{2}$ can react with the epoxy monomer. The slightly agglomerated MCNT- $\mathrm{NH}_{2}$ can realize the thermal conduction lap between the boron nitride sheets, which can be said to provide a thermal conduction path for the composite. MCNT-NH $\mathrm{N}_{2}$ plays a role in increasing the thermal conduction path of h-BN/LCERs composite and decreasing the large interface thermal resistance of fillers and resin matrix. Figure $4 c$ and Figure $4 d$ visually depicted the heat dissipation simulations of 50\% h-BN/ LCERs and 50\% h-BN@MCNT-NH $\mathrm{N}_{2} /$ LCERs composites, respectively. where the red rectangle represents the heating element, and the red line represents a thermal conductive path, MCNT$\mathrm{NH}_{2}$ plays a crucial role in the thermal conductivity of h-BN@MCNT-NH $\mathrm{N}_{2} /$ LECRs.

\subsection{Thermal conductivity of the composite}

The thermal conductivity of h-BN@MCNT- $\mathrm{NH}_{2} /$ LECRs and h-BN/LECRs were investigated with different loading of the filler at room temperature. As shown in Figure 5, When the content of the filler is the same, h-BN@MCNT-NH ${ }_{2} /$ LECR has a higher thermal conductivity than h-BN/LECR. In particular, h-BN@MCNT-NH $2 /$ LECRs having an h-BN@MCNT-NH ${ }_{2}$ content of 50 wt $\%$ has a high thermal conductivity of $2.775 \mathrm{~W} /(\mathrm{m} \cdot \mathrm{K})$ at room temperature, which is about 8.43 times that of a pure LCERs. While the thermal conductivity of h-BN/LECRs with $50 \mathrm{wt} \% \mathrm{~h}-\mathrm{BN}$ only reached $2.169 \mathrm{~W} /(\mathrm{m} \cdot \mathrm{K})$. This is due to the fact that the heat is transmitted by the vibrational modes of atoms, called phonons, with a dual character particle-wave, and the resin and h-BN are two different substances, one is crystalline, and the other is amorphous, phonon scattering easily occurs at the boundary between resin and h-BN. However, MCNT-NH $\mathrm{N}_{2}$ in the h-BN@MCNT- $\mathrm{NH}_{2} / \mathrm{LCERs}$ plays a role in increasing the thermal conduction path of h-BN/LCERs and decreasing the large interface thermal resistance of fillers and resin matrix. This makes h-BN@MCNT$\mathrm{NH}_{2} /$ LCERs composites have higher thermal conductivity than h-BN/LCERs.

Different theoretical models have been developed to study the thermal conductivity of composite 

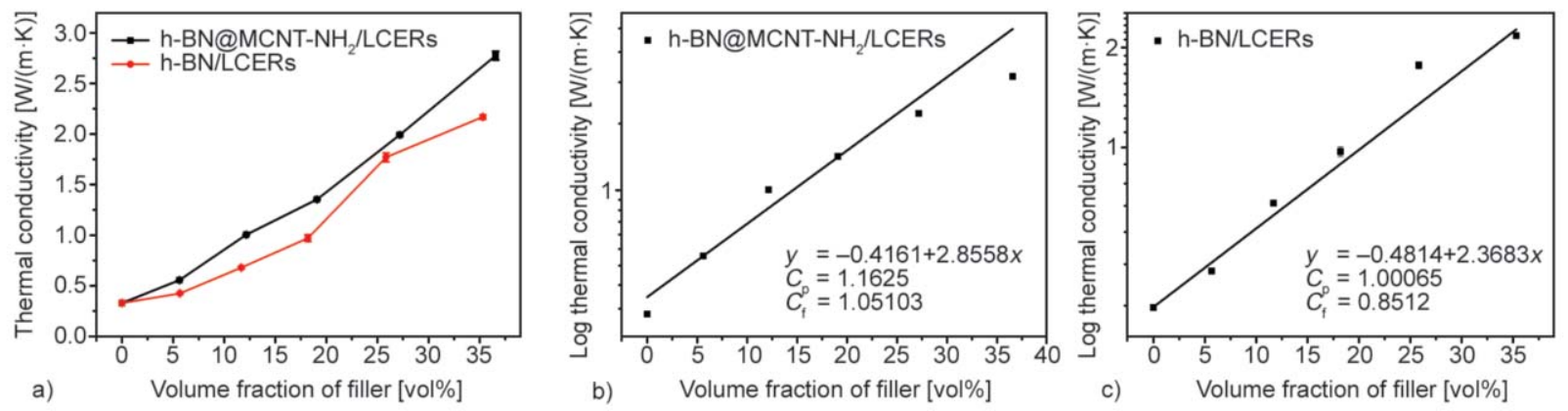

Figure 5. (a) Thermal conductivity of h-BN@MCNT-NH $2 /$ LECRs and h-BN/LECRs vs. the volume fraction of filler, (b) and (c) represent the logarithmic values of the thermal conductivity of h-BN@MCNT-NH$/ 2 E C R s$ and h-BN/LECRs as a function of volume fraction the filler, respectively.

materials, including Hatta-Taya's model [31, 32], Maxwell-Eucken's model [33], Agari's model [2, 34] and Bruggeman's model [35]. The factors that should be considered when choosing a theoretical model, such as particle size or shape, solids loading, and homogeneity of the dispersed phase in the resin matrix [33]. Assuming the heat conduction of the h-BN@MCNT-NH ${ }_{2} /$ LECRs and h-BN/LECRs composites are in an ideal condition, which is independent of isotropic and anisotropic properties. We chose the Agari's model to estimate the thermal conductivity of the composites. Agari's model was confirmed to be adaptable to thermal conductivities of varieties of polymer composite systems filled with spherical or irregular fillers [34]. Its mathematical expression is given by Equation (1):

$$
\log \lambda_{\mathrm{c}}=C_{\mathrm{f}} \cdot \log \left(\frac{\lambda_{\mathrm{f}}}{C_{\mathrm{p}} \cdot \lambda_{\mathrm{p}}}\right) \cdot V_{\mathrm{f}}+\log \left(C_{\mathrm{p}} \cdot \lambda_{\mathrm{p}}\right)
$$

where $\lambda_{\mathrm{c}}$ is the thermal conductivity of a composite; $\lambda_{\mathrm{f}}$ is the thermal conductivity of fillers $(200 \mathrm{~W} /(\mathrm{m} \cdot \mathrm{K}))$ [36]; $\lambda_{\mathrm{p}}$ is the thermal conductivity of polymer resin $(0.33 \mathrm{~W} /(\mathrm{m} \cdot \mathrm{K})) ; V_{\mathrm{f}}$ is the volume fraction of fillers; $C_{\mathrm{p}}$ measures the impact of filler on the structure of the liquid crystal epoxy resin; and $C_{\mathrm{f}}$ evaluates whether the filler can form the thermal conductive chains easily. The higher the value of $C_{\mathrm{f}}$ suggests the easier formation of the conductive chains in epoxy resin matrix [37]. Thermal conductivity of composites with different contents was presented in Figure $5 \mathrm{a}$. After linearly fitting the thermal conductivity data to the volume fraction, as shown in Figure $5 \mathrm{~b}$ and $5 \mathrm{c}$, there is a certain error between the prediction of the Agari's model and the actual thermal conductivity of h-BN/LECR and h-BN@MCNT-NH $2 /$ LECRs, but it is generally acceptable. In Xiao's article, when using Agari's model to simulate the thermal conductivity of silicon carbide nanowires $(\mathrm{SiCw}) /$ epoxy resin composite, there are multiple deviant points [38]. By Agari's model fitting of h-BN/LECR and h-BN@MCNT-NH $2 /$ LECRs, the corresponding value of $C_{\mathrm{p}}$ and $C_{\mathrm{f}}$ can be obtained as shown in Figure $5 \mathrm{~b}$ and $5 \mathrm{c}$. The value of $C_{\mathrm{p}}$ of h-BN@MCNT-NH $/$ LECRs and h-BN/LECRs is 1.1625 and 1.0006, respectively; The value of $C_{\mathrm{f}}$ of h-BN@MCNT- $\mathrm{NH}_{2} /$ LECRs and h-BN/LECR is 1.0510 and 0.8512 , respectively. The value of $C_{\mathrm{p}}$ of h-BN@MCNT- $\mathrm{NH}_{2} /$ LECRs is higher than of h-BN/LECRs, indicating that the hybrid filler has a greater impact on the polymer, which is attributed to the amino group in the filler participating in the curing process of epoxy resin. The value of $C_{\mathrm{f}}$ for the h-BN@MCNT-NH $/$ LECRs is larger than that of h-BN/LECR. It reveals that h-BN@MCNT-NH $\mathrm{N}_{2} /$ LCERs more easily formed thermal conductive paths in the resin matrix than h-BN/LCERs. We can seem to conclude that MCNT$\mathrm{NH}_{2}$ plays a role in increasing the thermal conduction path of h-BN/LCERs composite and decreasing the large interface thermal resistance of fillers and resin matrix.

\subsection{Thermal stability of the composites}

Thermal stability is one of the important properties for the composites in practical application. The thermal degradation behaviors of 50\% h-BN@MCNT$\mathrm{NH}_{2} /$ LCERs, 50\% h-BN/LCERs, 10\% h-BN@MCNT$\mathrm{NH}_{2} /$ LCERs, and $10 \%$ h-BN/LCER composites were investigated by TGA. The thermal stability of the composites was manifested in the temperature of thermal decomposition. The temperature value of $5 \%$ weight loss corresponds to at the temperature of the on-set decomposition reaction $[39,40]$. It can be seen from Figure $6 \mathrm{a}$ that the temperature of the on-set decomposition reaction of the composite is about 

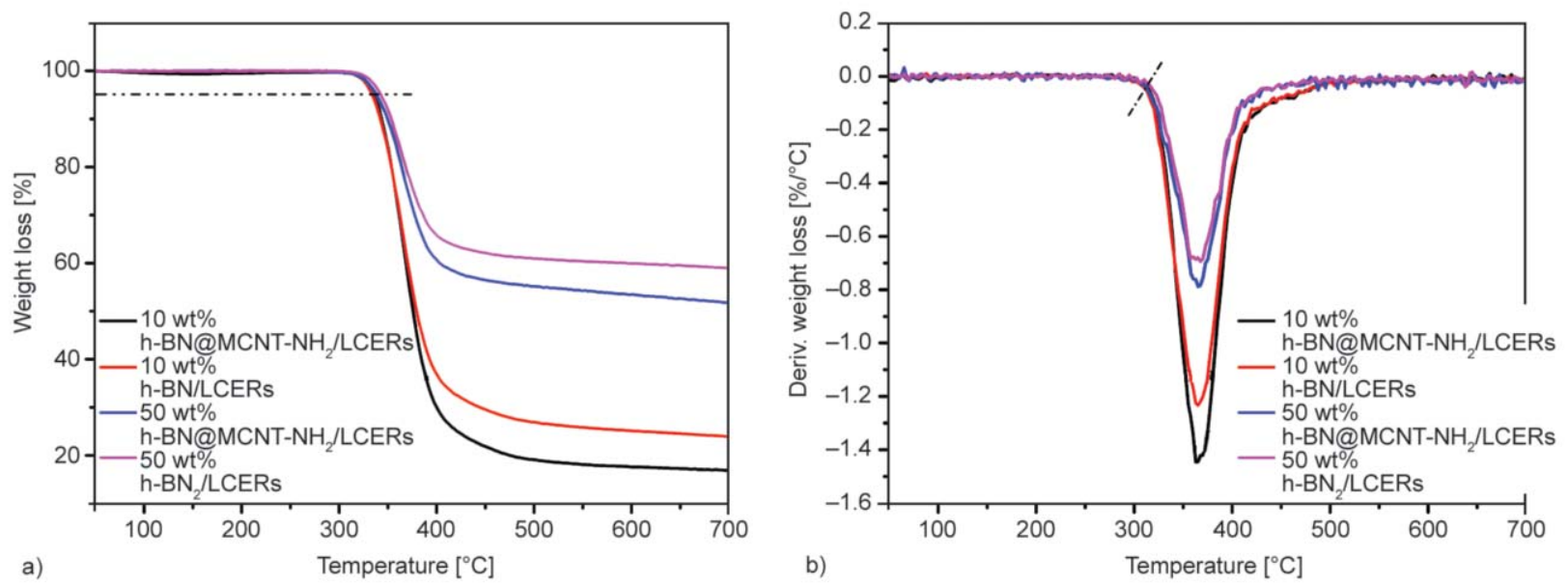

Figure 6. (a) TGA and (b) DTG curves of 50\% h-BN@MCNT-NH $2 /$ LCERs, 50\% h-BN/LCERs, 10\% h-BN@MCNT$\mathrm{NH}_{2} /$ LCERs, and $10 \% \mathrm{~h}-\mathrm{BN} / \mathrm{LCER}$ composites under nitrogen condition.

$330^{\circ} \mathrm{C}$. The residual weight of composites doesn't correspond to their content of 10 and $50 \mathrm{wt} \%$ at $700^{\circ} \mathrm{C}$, because the nitrogen atmosphere is used in the TGA test, the polymer forms carbon residues, and it is not completely decomposed [15]. Derivative thermogravimetric analysis (DTG) curve show weight loss rate of the composites. The absolute value of the curve represents the decomposition rate of the composite. It can be seen from Figure $6 \mathrm{~b}$ that the fastest thermal decomposition of 50 wt $\%$ h-BN@MCNT$\mathrm{NH}_{2} /$ LECRs and $50 \mathrm{wt} \% \mathrm{~h}-\mathrm{BN} /$ LECRs is slower than that of $10 \mathrm{wt} \%$ h-BN@MCNT-NH $2 /$ LECRs and $10 \mathrm{wt} \% \mathrm{~h}-\mathrm{BN} / \mathrm{LECRs}$. The possible reason is that the addition of $\mathrm{h}-\mathrm{BN}$ microplates temporarily blocked the heat that degrades the polymer matrix. Comparing the maximum decomposition rate of different types of fillers with the same content, h-BN@MCNT-NH ${ }_{2} /$ LECRs is faster than h-BN/ LECRs. This is because MCNT- $\mathrm{NH}_{2}$ having a nanometer size cannot prevent heat insulation and can transfer heat to the resin matrix more quickly.

\subsection{Application on electronic components}

At present, the heat-conducting substrate of commercial LED lamps is mostly metal aluminum, but aluminum has the characteristics of difficult to process and expensive. In order to practically evaluate the usability and thermal conductivity of h-BN@MCNT-NH $\mathrm{N}_{2} /$ LECR composites in LED lamps, we chose $3 \mathrm{~W}$ LED lamps and constant current converters(AC/DC) to build a conductive path with switches, as shown in Figure 7a. Composite materials with the same size (about $15 \mathrm{~mm} \times 15 \mathrm{~mm}$ $\times 2 \mathrm{~mm}$ ) were used as heat-transferring substrate material under the LED lamp. The surface temperature variations of the lighted LED lamp with time were recorded by an infrared thermal imager. The temperature of the LED lamp using 50\% h-BN@MCNT-NH $2 /$ LCERs, 50\% h-BN/LCERs, 10\% h-BN@MCNT-NH $2 /$ LCERs, and 10\% h-BN/ LCERs for heat dissipation was studied. It can be seen in Figure $7 \mathrm{~b}$ that, during the running process, the temperature of the LED lamp rises rapidly within 150 seconds after startup, and then the temperature is relatively constant from 200 to 600 seconds. The temperature using 50\% h-BN@MCNT-NH $2 /$ LCERs was eventually stabilized at $27.7^{\circ} \mathrm{C}$, below $50 \%$ h-BN/LCERs $\left(28.0^{\circ} \mathrm{C}\right), 10 \%$ h-BN@MCNT-NH ${ }_{2} /$ LCERs $\left(28.9^{\circ} \mathrm{C}\right), 10 \% \mathrm{~h}$-BN/LCERs $\left(29.5^{\circ} \mathrm{C}\right)$ and LCER $\left(30.6^{\circ} \mathrm{C}\right)$. This result illustrates that the $50 \%$ h-BN@MCNT-NH ${ }_{2} /$ LCERs composites have better heat-transferring capability, which is in good agreement with the order of thermal conductivity of the four samples. It is verified that the thermal conductivity of 50\% h-BN@MCNT-NH $2 /$ LCERs is higher in comparison with others. The above experimental results show that the 50\% h-BN@MCNT- $\mathrm{NH}_{2} /$ LCERs materials can guarantee timely dissipating of internally generated heat, resulting in increased lifetime and efficiency of the LED lamp eventually. Therefore, it is expected that 50\% h-BN@MCNT$\mathrm{NH}_{2} /$ LCER composites will be used in LED electronic products.

\section{Conclusions}

In this work, we introduced highly thermally conductive and fibrous MCNT-NH ${ }_{2}$ into h-BN/LCER composites to improve the thermal conductivity of 


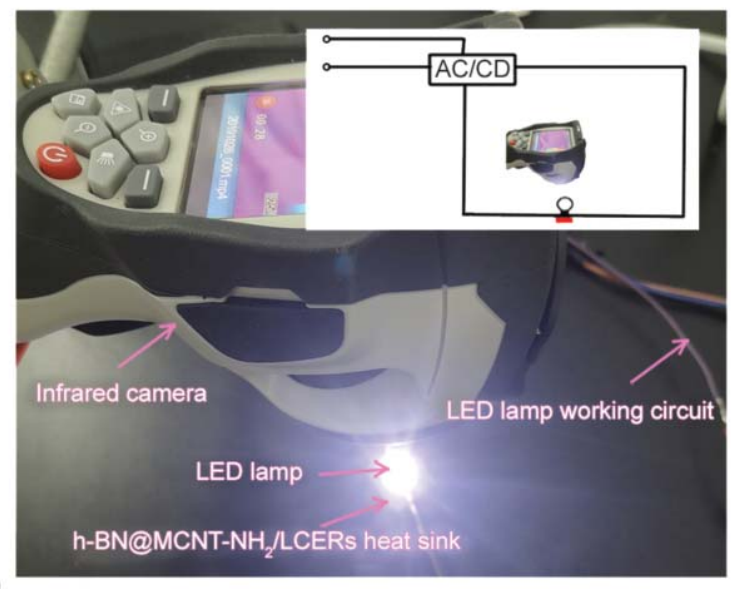

Figure 7. (a) Schematic diagram of h-BN@MCNT-NH $/$ LCERs composites been applied to LED and infrared camera been used to detect the heat of the surface temperature of LED lamps. (b) The surface temperature variations of the LED lamps against time.

the composites. First, we prepared h-BN@MCNT$\mathrm{NH}_{2}$ hybrid fillers. Then, the amino group in the hybrid filler participated in the curing process of the epoxy resin to prepare h-BN@MCNT-NH $2 /$ LCER composites. Subsequently, the effects of the same amount of h-BN@MCNT-NH $\mathrm{N}_{2}$ and h-BN were compared on the thermal conductivity of epoxy resins. The results showed that h-BN@MCNT-NH $/$ LECR had higher thermal conductivity. In order to explain the thermal conductivity of h-BN@MCNT- $\mathrm{NH}_{2}$ / LECR, we theoretically analyzed using the Agari's model and SEM to conclude that h-BN@MCNT$\mathrm{NH}_{2} /$ LCER compared to h-BN/LCER had more thermal conduction paths. The main reason is that the addition of MCNT- $\mathrm{NH}_{2}$ decreased the interface thermal resistance of h-BN and LCER. and made h-BN/ LCER form a thermally conductive chain. Finally, the usability and thermal conductivity of h-BN@MCNT$\mathrm{NH}_{2} /$ LECR composites was verified by LED lamps. The temperature of LED lamp using 50\% h-BN@MCNT-NH $2 /$ LCERs was eventually stabilized at $27.7^{\circ} \mathrm{C}$, it is expected that $50 \%$ h-BN@MCNT$\mathrm{NH}_{2} /$ LCER composites will be used in LED electronic products.

\section{Acknowledgements}

The authors would like to acknowledge the financial support from the National Key R\&D Program of China (No. 2017YFB0406200) and 'Major science and technology projects of Anhui Province’ (No.18030901083).

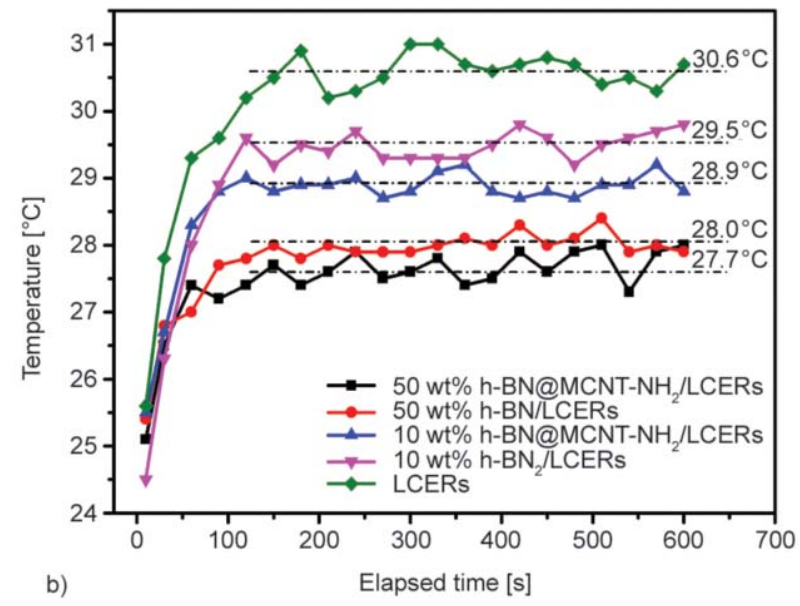

\section{References}

[1] Feng Y., Han G., Wang B., Zhou X., Ma J., Ye Y., Liu C., Xie X.: Multiple synergistic effects of graphene-based hybrid and hexagonal born nitride in enhancing thermal conductivity and flame retardancy of epoxy. Chemical Engineering Journal, 379, 122402/1-122402/13 (2020). https://doi.org/10.1016/j.cej.2019.122402

[2] Yeo H., Islam A. M., You N-H., Ahn S., Goh M., Hahn J. R., Jang S. G.: Characteristic correlation between liquid crystalline epoxy and alumina filler on thermal conducting properties. Composites Science and Technology, 141, 99-105 (2017).

https://doi.org/10.1016/j.compscitech.2017.01.016

[3] Chen H. Y., Ginzburg V. V., Yang J., Yang Y. F., Liu W., Huang Y., Du L. B., Chen B.: Thermal conductivity of polymer-based composites: Fundamentals and applications. Progress in Polymer Science, 59, 41-85 (2016). https://doi.org/10.1016/j.progpolymsci.2016.03.001

[4] Ha S. M., Lee H. L., Lee S-G., Kim B. G., Kim Y. S., Won J. C., Choi W. J., Lee D. C., Kim J., Yoo Y.: Thermal conductivity of graphite filled liquid crystal polymer composites and theoretical predictions. Composites Science and Technology, 88, 113-119 (2013). https://doi.org/10.1016/j.compscitech.2013.08.022

[5] Aradhana R., Mohanty S., Nayak S. K.: Novel electrically conductive epoxy/reduced graphite oxide/silica hollow microspheres adhesives with enhanced lap shear strength and thermal conductivity. Composites Science and Technology, 169, 86-94 (2019).

https://doi.org/10.1016/j.compscitech.2018.11.008

[6] Chen M., Hu W., Liang X., Zhang H., Zhang C., Song P., Zhang L., Li F., Chen F., Yang H.: The regulation of polymer structures and electro-optical properties of epoxy-mercaptan-based phase separated liquid crystals/ polymer composites. Polymer, 127, 1-7 (2017). https://doi.org/10.1016/j.polymer.2017.07.003 
[7] Li M., Tang C., Zhang L., Shang B., Zheng S., Qi S.: A thermally conductive and insulating epoxy polymer composite with hybrid filler of modified copper nanowires and graphene oxide. Journal of Materials Science: Materials in Electronics, 29, 4948-4954 (2018). https://doi.org/10.1007/s10854-017-8454-5

[8] Pan C., Kou K., Jia Q., Zhang Y., Wu G., Ji T.: Improved thermal conductivity and dielectric properties of $\mathrm{hBN} / \mathrm{PTFE}$ composites via surface treatment by silane coupling agent. Composites Part B: Engineering, 111, 83-90 (2017).

https://doi.org/10.1016/j.compositesb.2016.11.050

[9] Zhu H., Li Y., Fang Z., Xu J., Cao F., Wan J., Preston C., Yang B., Hu L.: Highly thermally conductive papers with percolative layered boron nitride nanosheets. ACS Nano, 8, 3606-3613 (2014). https://doi.org/10.1021/nn500134m

[10] Xiao C., Leng X., Zhang X., Zheng K., Tian X.: Improved thermal properties by controlling selective distribution of AlN and MWCNT in immiscible polycarbonate (PC)/Polyamide 66 (PA66) composites. Composites Part A: Applied Science and Manufacturing, 110, 133-141 (2018).

https://doi.org/10.1016/j.compositesa.2018.03.030

[11] Yao Y., Zeng X., Pan G., Sun J., Hu J., Huang Y., Sun R., Xu J-B., Wong C-P.: Interfacial engineering of silicon carbide nanowire/cellulose microcrystal paper toward high thermal conductivity. ACS Applied Materials and Interfaces, 8, 31248-31255 (2016).

https://doi.org/10.1021/acsami.6b10935

[12] Kang D-G., Kim N., Park M., Nah C., Kim J. S., Lee C-R., Kim Y., Kim C. B., Goh M., Jeong K-U.: Interfacial engineering for the synergistic enhancement of thermal conductivity of discotic liquid crystal composites. ACS Applied Materials and Interfaces, 10, 31553159 (2018).

https://doi.org/10.1021/acsami.7b16921

[13] Prolongo S. G., Meliton B. G., Del Rosario G., Ureña A.: New alignment procedure of magnetite-CNT hybrid nanofillers on epoxy bulk resin with permanent magnets. Composites Part B: Engineering, 46, 166-172 (2013).

https://doi.org/10.1016/j.compositesb.2012.10.002

[14] Wu H., Kessler M. R.: Multifunctional cyanate ester nanocomposites reinforced by hexagonal boron nitride after noncovalent biomimetic functionalization. ACS Applied Materials and Interfaces, 7, 5915-5926 (2015). https://doi.org/10.1021/acsami.5b00147

[15] Yu B., Xing W., Guo W., Qiu S., Wang X., Lo S., Hu Y.: Thermal exfoliation of hexagonal boron nitride for effective enhancements on thermal stability, flame retardancy and smoke suppression of epoxy resin nanocomposites via sol-gel process. Journal of Materials Chemistry A, 4, 7330-7340 (2016).

https://doi.org/10.1039/c6ta01565d
[16] Yuan C., Duan B., Li L., Xie B., Huang M., Luo X.: Thermal conductivity of polymer-based composites with magnetic aligned hexagonal boron nitride platelets. ACS Applied Materials and Interfaces, 7, 13000-13006 (2015).

https://doi.org/10.1021/acsami.5b03007

[17] Shen H., Guo J., Wang H., Zhao N., Xu J.: Bioinspired modification of h-BN for high thermal conductive composite films with aligned structure. ACS Applied Materials and Interfaces, 7, 5701-5708 (2015).

https://doi.org/10.1021/am507416y

[18] Zhang D-L., Zha J-W., Li W-K., Li C-Q., Wang S-J., Wen Y., Dang Z-M.: Enhanced thermal conductivity and mechanical property through boron nitride hot string in polyvinylidene fluoride fibers by electrospinning. Composites Science and Technology, 156, 1-7 (2018). https://doi.org/10.1016/j.compscitech.2017.12.008

[19] Terao T., Bando Y., Mitome M., Zhi C., Tang C., Golberg D.: Thermal conductivity improvement of polymer films by catechin-modified boron nitride nanotubes. The Journal of Physical Chemistry C, 113, 13605-13609 (2009). https://doi.org/10.1021/jp903159s

[20] Qin T., Wang H., He J., Wang R., Qu Q., Tian X.: Preparation and application of high thermal conductivity TMBPDGE-DDM@h-BN composites. Materials Research Express, 6, 095320/1-095320/10 (2019). https://doi.org/10.1088/2053-1591/ab3003

[21] Wang Z-G., Huang Y-F., Zhang G-Q., Wang H-Q., Xu J-Z., Lei J., Zhu L., Gong F., Li Z-M.: Enhanced thermal conductivity of segregated poly(vinylidene fluoride) composites via forming hybrid conductive network of boron nitride and carbon nanotubes. Industrial and Engineering Chemistry Research, 57, 10391-10397 (2018).

https://doi.org/10.1021/acs.iecr.8b01764

[22] Zhang X., Zhang J., Li C., Wang J., Xia L., Xu F., Zhang X., Wu H., Guo S.: Endowing the high efficiency thermally conductive and electrically insulating composites with excellent antistatic property through selectively multilayered distribution of diverse functional fillers. Chemical Engineering Journal, 328, 609-618 (2017). https://doi.org/10.1016/j.cej.2017.07.087

[23] Shi Q., Zhu A.: Interface regulation of graphene/carbon nanotube on the thermal conductivity and anticorrosion performance of their nanocomposite. Progress in Organic Coatings, 140, 105480/1-105480/7 (2020).

https://doi.org/10.1016/j.porgcoat.2019.105480

[24] Kim S. Y., Jang J-U., Haile B. F., Lee M. W., Yang B.: Swarm intelligence integrated micromechanical model to investigate thermal conductivity of multi-walled carbon nanotube-embedded cyclic butylene terephthalate thermoplastic nanocomposites. Composites Part A: Applied Science and Manufacturing, 128, 105646/1105646/8 (2020). https://doi.org/10.1016/j.compositesa.2019.105646 
[25] Guo H., Wang Q., Liu J., Du C., Li B.: Improved interfacial properties for largely enhanced thermal conductivity of poly(vinylidene fluoride)-based nanocomposites via functionalized multi-wall carbon nanotubes. Applied Surface Science, 487, 379-388 (2019). https://doi.org/10.1016/j.apsusc.2019.05.070

[26] Yan F., Liu L., Li M., Zhang M., Shang L., Xiao L., Ao Y.: One-step electrodeposition of $\mathrm{Cu} / \mathrm{CNT} / \mathrm{CF}$ multiscale reinforcement with substantially improved ther$\mathrm{mal} /$ electrical conductivity and interfacial properties of epoxy composites. Composites Part A: Applied Science and Manufacturing, 125, 105530/1-105530/10 (2019). https://doi.org/10.1016/j.compositesa.2019.105530

[27] Su Z., Wang H., Ye X., Tian K., Huang W., He J., Guo Y., Tian X.: Anisotropic thermally conductive flexible polymer composites filled with hexagonal born nitride (h-BN) platelets and ammine carbon nanotubes (CNT$\mathrm{NH}_{2}$ ): Effects of the filler distribution and orientation. Composites Part A: Applied Science and Manufacturing, 109, 402-412 (2018). https://doi.org/10.1016/j.compositesa.2018.03.021

[28] Lu F., Wang F., Gao W., Huang X., Zhang X., Li Y. Aqueous soluble boron nitride nanosheets via anionic compound-assisted exfoliation. Materials Express, 3, 144-150 (2013).

https://doi.org/10.1166/mex.2013.1110

[29] Muhabie A. A., Cheng C-C., Huang J-J., Liao Z-S., Huang S-Y., Chiu C-W., Lee D-J.: Non-covalently functionalized boron nitride mediated by a highly self-assembled supramolecular polymer. Chemistry of Materials, 29, 8513-8520 (2017).

https://doi.org/10.1021/acs.chemmater.7b03426

[30] Ding J-H., Zhao H-R., Yu H-B.: High-yield synthesis of extremely high concentrated and few-layered boron nitride nanosheet dispersions. 2D Materials, 5, 045015/1-045015/7 (2018).

https://doi.org/10.1088/2053-1583/aad51a

[31] Tarani E., Terzopoulou Z., Bikiaris D. N., Kyratsi T., Chrissafis K., Vourlias G.: Thermal conductivity and degradation behavior of HDPE/graphene nanocomposites. Journal of Thermal Analysis and Calorimetry, 129, 1715-1726 (2017).

https://doi.org/10.1007/s10973-017-6342-0

[32] Ghebrid N., Guellal M., Rouabah F.: Thermal conductivity of polymer composite pigmented with titanium dioxide. Applied Physics A, 123, 276/1-276/8 (2017). https://doi.org/10.1007/s00339-017-0889-2
[33] Yan C., Yu T., Ji C., Zeng X., Lu J., Sun R., Wong C-P.: $3 \mathrm{D}$ interconnected high aspect ratio tellurium nanowires in epoxy nanocomposites: Serving as thermal conductive expressway. Journal of Applied Polymer Science, 136, 47054/1-47054/7 (2019). https://doi.org/10.1002/app.47054

[34] Agari Y., Ueda A., Nagai S.: Thermal conductivity of a polymer composite. Journal of Applied Polymer Science, 49, 1625-1634 (1993). https://doi.org/10.1002/app.1993.070490914

[35] Boudenne A., Ibos L., Fois M., Gehin E., Majeste J-C.: Thermophysical properties of polypropylene/aluminum composites. Journal of Polymer Science Part B: Polymer Physics, 42, 722-732 (2004). https://doi.org/10.1002/polb.10713

[36] Hu J., Huang Y., Yao Y., Pan G., Sun J., Zeng X., Sun R., Xu J-B., Song B., Wong C-P.: Polymer composite with improved thermal conductivity by constructing a hierarchically ordered three-dimensional interconnected network of BN. ACS Applied Materials and Interfaces, 9, 13544-13553 (2017). https://doi.org/10.1021/acsami.7b02410

[37] Gu J., Lv Z., Wu Y., Zhao R., Tian L., Zhang Q.: Enhanced thermal conductivity of $\mathrm{SiCp} / \mathrm{PS}$ composites by electrospinning-hot press technique. Composites Part A: Applied Science and Manufacturing, 79, 8-13 (2015). https://doi.org/10.1016/j.compositesa.2015.09.005

[38] Xiao C., Chen L., Tang Y., Zhang X., Zheng K., Tian X.: Enhanced thermal conductivity of silicon carbide nanowires $(\mathrm{SiCw}) /$ epoxy resin composite with segregated structure. Composites Part A: Applied Science and Manufacturing, 116, 98-105 (2019).

https://doi.org/10.1016/j.compositesa.2018.10.023

[39] Song J., Dai Z., Li J., Tong X., Zhao H.: Polydopaminedecorated boron nitride as nano-reinforcing fillers for epoxy resin with enhanced thermomechanical and tribological properties. Materials Research Express, 5, 075029/1-075029/10 (2018). https://doi.org/10.1088/2053-1591/aab529

[40] Xu M., Lei Y., Ren D., Chen S., Chen L., Liu X.: Synergistic effects of functional CNTs and h-BN on enhanced thermal conductivity of epoxy/cyanate matrix composites. Nanomaterials, 8, 997/1-997/13 (2018). https://doi.org/10.3390/nano8120997 